\title{
EFFECTS OF CENTELLA ASIATICA (L.) LEAF EXTRACT ON BONE CALCIUM AND PHOSPHATE LEVELS OF OVARIECTOMIZED RATS
}

\section{YULITA BONG, SRI ANGKY SOEKANTO*, ERIK IDRUS}

\author{
Department of Oral Biology, Faculty of Dentistry, Universitas Indonesia, Jakarta, Indonesia. Email: sasoekanto@gmail.com
}

Received 14 October 2018, Revised and Accepted 15 January 2019

ABSTRACT

Objective: This study aimed to evaluate the efficacy of Centella asiatica leaf extract in maintaining calcium and phosphate levels in the bone for preventing osteoporosis in postmenopausal women.

Methods: The obtained C. asiatica leaf extract was administered to ovariectomized rats at the doses of 60,120 , and 180 mg/kg body weight (BW) for 30 days. Subsequently, the rats were euthanized by ether overdose, and bone calcium and phosphate levels were determined by the wet ashing technique and spectrophotometry.

Results: The results showed no significant differences in the bone calcium and phosphate levels among rats administered with different doses of C. asiatica leaf extract.

Conclusion: Our results indicate that $C$. asiatica leaf extract cannot be used as a source of phytoestrogens to maintain calcium and phosphate levels in the bones.

Keywords: Bone, calcium, Centella asiatica, Phytoestrogen, Osteoporosis.

(C) 2019 The Authors. Published by Innovare Academic Sciences Pvt Ltd. This is an open access article under the CC BY license (http://creativecommons. org/licenses/by/4. 0/) DOI: http://dx.doi.org/10.22159/ijap.2019.v11s1.AR169

\section{INTRODUCTION}

Osteoporosis is a chronic and progressive disease characterized by low bone mass, bone microstructure disorder, and decreased bonestrength that causes brittleness and susceptibility to bone fracture [1]. It is associated with old age and has become a major health problem worldwide, including in developed countries, especially in postmenopausal women [2].

Bone remodeling is a process that maintains bone health by removing old bone cells and consistently replacing them with new ones. During menopause, bone resorption increases and causes an imbalance resulting in skeletal abnormalities, which increase the risk of fractures [2]. Decreased estrogen levels increase serum cytokine levels that lead to osteoclast formation and reactions in the trabecular bone, resulting in bone resorption [3].

Two types of cytokines are produced by bone marrow stromal cells and osteoblasts that are essential in osteoclastogenesis induced by macrophage colony-stimulating factor and receptor activator of nuclear factor kappa B ligand (RANKL), which are members of the tumor necrosis factor (TNF) superfamily. RANKL is expressed by mononuclear osteoclast precursors that play a role in osteoclast formation, whereas osteoprotegerin (OPG) is produced by stromal and osteoblast cells and is a natural inhibitor of RANKL and, therefore, antagonistic to the osteoclastogenic activity of RANKL [4].

Estrogen works by activating the Fas ligand that binds to estrogen receptors on the target cell [5]. Estrogen affects skeletal and extracellular activities. Skeletal activity can be direct and indirect. Direct skeletal activity is associated with estrogen receptors in osteoblasts and osteoclasts, whereas indirect activity is associated with estrogen receptors in other cells including stromal cells that regulate OPG and immune cells that affect bone homeostasis [4].

Estrogen deficiency in postmenopausal women results in increased RANKL expression in bone marrow cells, which increase osteoclast formation, leading to increased bone resorption. In addition, when estrogen is deficient, the stimulation of OPG formation in osteoblasts that create an antiresorptive effect on bone ceases [6].

Extracellular activity in estrogen deficiency increases calcium excretion in the kidneys and decreases calcium absorption in the intestines. Estrogen deficiency also causes increased parathyroid hormone levels as a compensation for the loss of calcium in the body. In addition, it is known that the increased production of various inflammatory cytokines and mediators, such as interleukin (IL)-1, IL-6, TNF- $\alpha$, and prostaglandin E2, affects the pathogenesis of osteoporosis because it increases RANKL expression. Estrogen deficiency also affects cytokine function in the immune system. In addition, our study results showed that estrogen deficiency affects the activation of T-cells, which are also involved in the pathogenesis of osteoporosis [4].

Hormone replacement therapy is the only treatment that shows concrete results in terms of preventing the loss of bone mass density and reducing the risk of fracture in osteoporosis patients [2]. However, further, research on the long-term effects of hormone replacement therapy revealed that hormone therapy increases the risk of breast cancer, heart disease, thrombosis, and stroke. Therefore, this therapy has been discontinued because the risks exceed the benefits [7].

The word phytoestrogen is derived from "phyto" meaning plant because they are found in plants, and "estrogen," due to their ability to function like naturally produced estrogen in the human body [8]. These compounds can bind to estrogen receptors in the body and can exhibit estrogen-like effects on the target tissues; therefore, they can be used as a substitute for estrogen in postmenopausal women in the form of a dietary supplement. Four types of compounds belong to this class of phytoestrogens, namely isoflavonoids, flavonoids, stilbenes, and lignans $[8,9]$.

Centella asiatica (also known as gotu kola and Hydrocotyle asiatica) is an herb with kidney-shaped leaves found in India, Sri Lanka, Madagascar, 
South Africa, Australia, China, and Japan [10]. It is used as a traditional medicine to treat various diseases [11]. Its pythritic leaves contain terpenoids, flavonoids, olive oil, and amino acids. Flavonoids found in C. asiatica include quercetin, kaempferol, catechin, rutin, naringin [6], apigenin, luteolin [12], castilliferol, and castillicetin [13]. It is known that several flavonoids found in $C$. asiatica are phytoestrogens that are beneficial for the bone health [14].

Quercetin and kaempferol are flavonols and phytoestrogens that can increase estrogen-like activity at certain doses $[14,15]$. In vitro quercetin and kaempferol not only inhibit bone resorption but also directly induce osteoclast apoptosis at certain doses. However, the mechanism underlying the induction of osteoclast apoptosis is difficult to uncover because at lower doses, these flavonoids, especially kaempferol, can even act as pro-oxidants. Several studies involving ovariectomized rats have also reported a significant decrease in bone resorption after quercetin administration [16].

Meanwhile, studies investigating estrogen deficiency in mice have shown that catechins increase osteoblast activity [17]. Catechins can suppress bone resorption and may be used as for treating osteoporosis [18]. Rutin may prevent osteopenia in ovariectomized rats [19], and certain doses of naringin can stimulate osteogenic activity in the line of osteoblasts and bone mesenchymal stem cells. Naringin has recently been shown to suppress the osteoclastogenic activity associated with IL-1 [20].

Apigenin has antioxidant, antitumor, and anti-inflammatory properties and inhibits the production of inflammatory mediators such as IL-6, TNF- $\alpha$, and IL-8 [21]. The flavonoid luteolin is an important antioxidant, a free radical antagonist, an anti-inflammatory agent, and an immune system modulator. Research has shown that luteolin may inhibit TNF- $\alpha$ formation [22].

Building on the previous studies on the properties of compounds found in $C$. asiatica, the present study aimed to evaluate the efficacy of $C$. asiatica leaf extract in maintaining calcium and phosphate levels in the bone for preventing osteoporosis in postmenopausal women, as well as to determine the optimal dose.

\section{METHODS}

The study protocol had been approved by the Dental Research Ethics Committee, Faculty of Dentistry, Universitas Indonesia. In the experimental laboratory study, 30 rats were equally divided (five rats each) into the following six test groups: Sham, control $(-)$, control $(+)$, and treatment with $C$. asiatica leaf extract at doses of 60,120 , and $180 \mathrm{mg} / \mathrm{kg}$ BW. All rats (except sham) were ovariectomized and treated for 30 days. Sham and control (-) rats were given distilled water, whereas control $(+)$ rats were given Vitamin $\mathrm{E}$.

C. asiatica leaf extract was obtained by drying, simplicia, immersion in alcohol, filtration, and evaporation to produce a thick extract. The extract was then dissolved in distilled water and administered to the rats daily through a feeding tube at the prescribed dose. Vitamin E was dissolved in vegetable oil and administered to control (+) animals through a feeding tube at a dose of $60 \mathrm{mg} / \mathrm{kg} \mathrm{BW}$.

On day 31, all rats were euthanized by ether overdose, and their thighs were removed and cleansed to obtain the femur and dry tibia. Calcium levels in the left femur and phosphate levels in the right femur were determined by the wet destruction technique followed by spectrophotometric analysis.

Calcium and phosphate level test results were analyzed by the Shapiro-Wilk normality test. Normally distributed data on calcium levels were then tested by one-way analysis of variance (ANOVA) and the Pearson correlation test. Data on phosphate levels were not normally distributed and were, therefore, tested by the non-parametric Kruskal-Wallis test and the Spearman correlation test. A significance level of $0.05(p=0.05)$ and a confidence level of $95 \%(\alpha=0.05)$ were considered in the statistical tests.

\section{RESULTS}

The measured calcium and phosphate levels in each group are presented in Figs. 1 and 2, respectively. One-way ANOVA revealed no significant difference in calcium levels among the treatment groups. The Pearson correlation test demonstrated a very weak and insignificant increase in calcium levels in the treatment groups compared with that in the

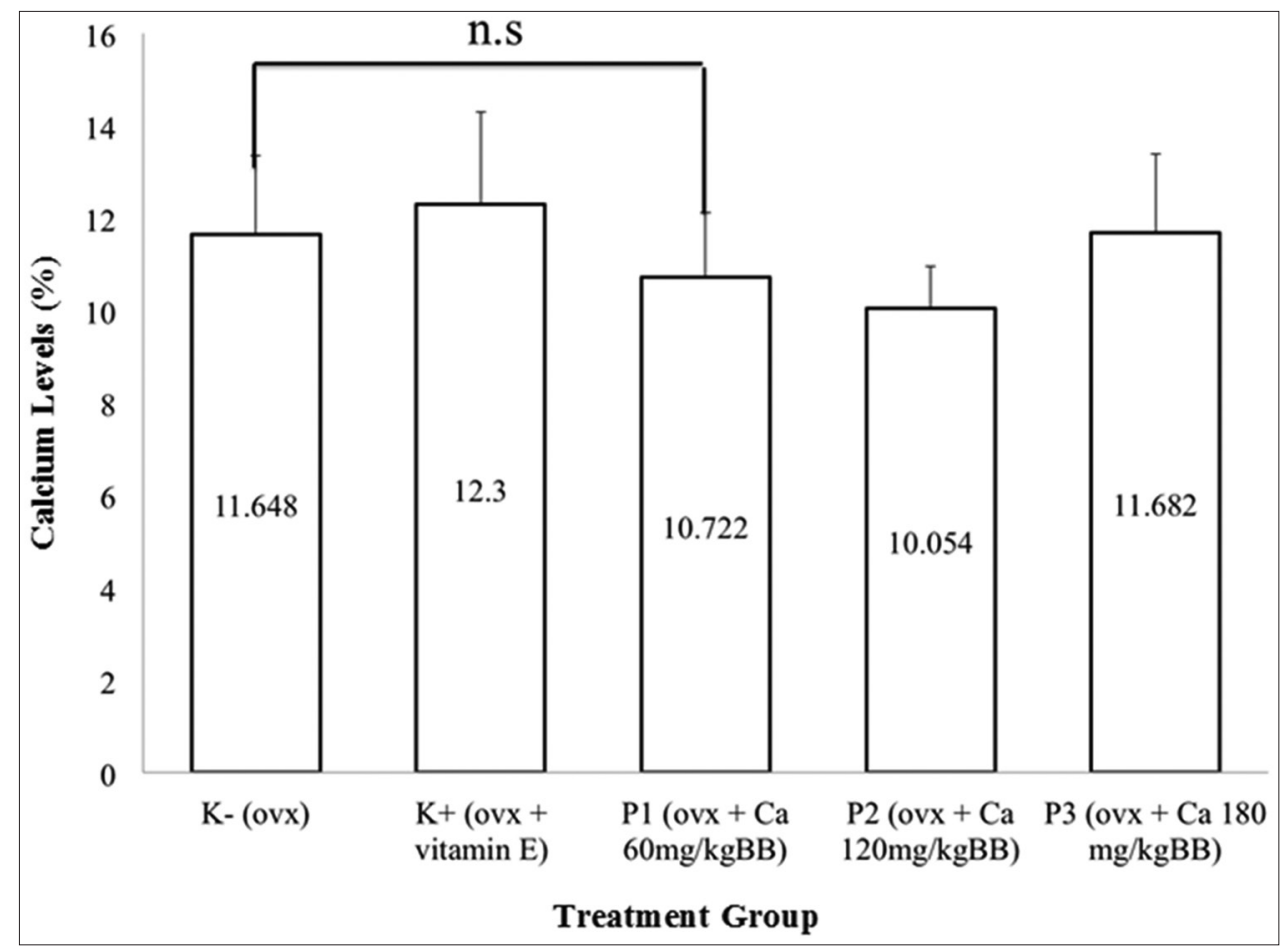

Fig. 1: Mean femur bone calcium levels 


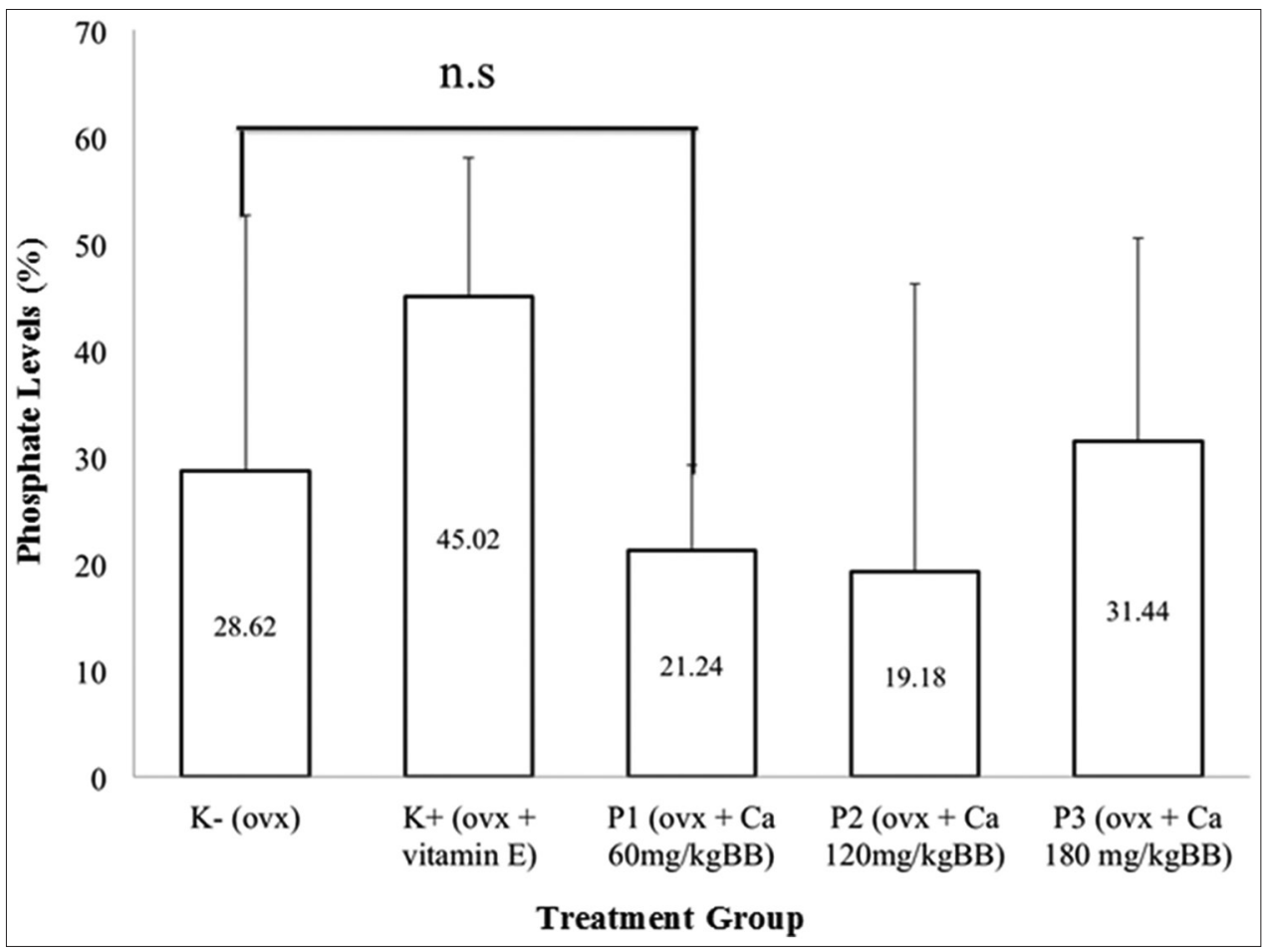

Fig. 2: Mean femur bone phosphate levels

other groups. The Kruskal-Wallis test revealed no significant difference in phosphate levels among the treatment groups. The Spearman test detected an insignificant increase in phosphate levels in the treatment groups compared with that in the other groups.

\section{DISCUSSION}

Our results demonstrated no significant difference in the calcium and phosphate levels among the treatment groups. Although the correlation test showed a very weak correlation between the dose of $C$. asiatica leaf extract and calcium levels and a stronger correlation between the dose of $C$. asiatica leaf extract and phosphate levels, both correlations were insignificant. This is contradictory to the previous studies arguing that phytoestrogens may prevent bone resorption [9].

Several factors may explain why the results of this study are not in accordance with the findings of the previous studies: The mechanism of dose-dependent phytoestrogens, time required for osteoclasts to break the mineral crystals in bone, high bone mass, uncontrolled feeding of mice, and inaccuracy factors in the calculations.

Each phytoestrogen has specific active constituents that exert their function at different doses [23]. At certain doses, some phytoestrogens, such as kaempferol and quercetin, may function as pro-oxidant agents, causing oxidative stress that can increase osteoclast activity resulting in bone resorption [16]. Many studies have been conducted with the aim of identify the working dose of phytoestrogens, which have not yet been determined due to their complex actions [23].

C. asiatica contains unknown concentrations of several types of the phytoestrogens. In this study, the doses present in C. asiatica leaf extract may not have provided the desired effect. It is also possible that these individual phytoestrogens exert their function at different doses; therefore, the bone calcium and phosphate levels of ovariectomized rats were not significantly different from those of rats that were not administered $C$. asiatica extract. In addition, because of dose-dependent action of phytoestrogens, the different concentrations of $C$. asiatica extract might have failed to have affect bone calcium and phosphate levels.
Osteoclasts complete bone resorption in approximately 3 weeks [24]. In this study, the treatment time from ovariectomy to euthanasia was 30 days, so bone mass changes were less visible. Moreover, the femur bone consists mainly of cortical bone that is slower to resorb than the trabecular bone [6,25]. Studies have shown that a significant increase in the femur mass attributed to catechins lasted for 3 months [17]. Our study period was not sufficient to show differences in calcium and phosphate levels in the rat femur bone.

One of the important factors in maintaining bone density is the iliac crest bone mass [26], which is influenced by race, age, gender, genetics, physical activity, and nutrient intake, especially that of calcium and Vitamin D [27]. Several factors that could affect this bone mass were not controlled in this study, such as physical activity and nutrient intake. The rats were left free in the cage, and each rat had a different level of activity. The amount of food intake of the rats was not controlled either, so the nutrient intake of each rat would also have been different depending on their appetite. Therefore, the highest bone mass of any uncontrolled rat would have affected the bone calcium and phosphate density, resulting in insignificant research results.

Natural products offer a rich source of structurally diverse substances with a wide range of biological activities, which could be useful for the development of alternative or adjunctive for osteoporosis therapies [28]. The market for anti-aging products is also growing gradually, and customers are aware of active principles in the cosmetics [29]. C. asiatica as natural product also can be an alternative treatment for osteoporosis in aging women.

\section{CONCLUSION}

Based on the results of this study, it can be concluded that $C$. asiatica leaf extract as a source of phytoestrogens could not help to maintain calcium and phosphate levels in the bones of ovariectomized rats. In addition, no difference was found among different concentrations of C. asiatica leaf extract in terms of their effects on the bone calcium and phosphate content in the ovariectomized rats. 


\section{CONFLICTS OF INTEREST}

There are no conflicts of interest to declare.

\section{REFERENCES}

1. Mauck K, Clarke B. Concise review for clinicians diagnosis, screening, prevention, and treatment of osteoporosis. Mayo Clin Pro 2006;81:662-72.

2. Ismal NM. Postmenopausal osteoporosis: Epidemiology, pathophysiology and treatment. Malays J Pathol 1997;19:21-5.

3. Simon L. Osteoporosis. Rheum Dis Clin N Am 2007;33:149-76.

4. Sipos W, Peter P, Martina R. Pathophysiology of osteoporosis. Themenschwerpunkt 2009;159:230-4.

5. Nakamura T, Imai Y, Matsumoto T, Sato S. Estrogen prevents bone loss via estrogen receptor $\alpha$ and induction of Fas ligand in osteoclasts. Cell 2007;130:811-23.

6. Zheng C, Qin L. Chemical components of Centella asiatica and their bioactivities. J Chin Integr Med 2007;5:348-51.

7. US Department of Health and Human Services. Facts about Menopausal Hormone Therapy. Available from: http://www.nhlbi.nih.gov/health/ women/pht facts.pdf. [Last accessed on 2013 Sep 20].

8. Dixon RA. Phytoestrogens. Annu Rev Plant Biol 2004;55:225-61.

9. Athena Urology and Urogynecology. What is Phytoestrogen. Available from: http://www.athena-urology.com/patient-docs/Phytoestrogen-seattlefemale-urologists-urogynecologists.pdf. [Last accessed on 2013 Sep 20].

10. Barnes J, Anderson L, Phillipson J. Herbal Medicines. Pharm Press 2007;3:71-3

11. Winarto WP, Surbakti M. The Efficacy and Use of Centella asiatica. Jakarta: Agro Media Pustaka; 2005.

12. Roy D, Barman S, Shalk M. Current updates on Centella asiatica L.: Phytochemistry, pharmacology and traditional uses. Med Plant Res 2013;3:4.

13. Subban R, Veerakumar A, Manimaran R, Hashim KM, Balachandran I. Two new flavonoids from Centella asiatica (Linn.). J Nat Med 2008;62:369-73.

14. Wong R, Rabie A. Effect of quercetin on preosteoblast and bone defects. Open Orthop J 2008;2:27-32

15. Guo AJ, Choi RC, Zheng KY, Chen VP, Dong TT, Wang ZT, et al. Kaempferol as a flavonoid induces osteoblastic differentiation via estrogen receptor signaling. Chin Med 2012;7:10.

16. Wattel A, Kamel S, Mentaverri R, Lorget F, Prouillet C, Petit JP, et al.
Potent inhibitory effect of naturally occuring flavonoids quercetin and kaempferol on in vitro osteoclastic bone resorption. Biochem Pharm 2003;65:35-42.

17. Bakhsh A, Mustapha NM, Mohamed S. Catechin-rich oil palm leaf extract enhances bone calcium content of estrogen-deficient rats. Nutrition 2013;29:667-72.

18. Kamon M, Zhao R, Sakamoto K. Green tea polyphenol (-) epigallocatechin gallate suppressed the differentiation of murine osteoblastic MC3T3-E1 cells. Cell Biol Int 2010;34:109-16.

19. Horcajada-Molteni M, Crespy V, Coxam V, Davicco MJ, Rémésy C, Barlet JP. Rutin inhibits ovariectomy-induced osteopenia in rats. J Bone Miner Res 2000;15:2251-8.

20. Hirata M, Matsumoto C, Morichika T, Miyaura C, Inada M. Naringin suppresses osteoclast formation and enhance bone mass in mice. J Health Sci 2009;55:463-7.

21. Wang J, Liao Y, Fan J, Ye T, Sun X, Dong S. Apigenin inhibits the expression of IL-6, IL-8, and ICAM-1 in DEHP-stimulated human umbilical vein endothelial cells in vivo. Inflammation 2012;35:1466-76.

22. Bhavna D, Jyoti K. Centella asiatica L.: The elixir of life. Int J Res Ayurveda Pharm 2011;2:431-8.

23. Umland EM, Cauffield JS, Kirk JK, Thomason TE. Phytoestrogens as therapeutic alternatives to traditional hormone replacement in postmenopausal women. Pharmacotherapy 2000;20:981-90.

24. Helmberg A. Bone Metabolism 2009-2010. p. 13. Available from: http://www.helmberg.at/bone-metabolism.htm. [Last accessed on 2000 Aug 20].

25. University of Cambridge. Structure and Composition of Bone 20042013. Available from: http://www.doitpoms.ac.uk/tlplib/bones/ structure.php[Last accessed on 2013 Nov 01]

26. Borer K. Physical activity in prevention and amelioration of osteoporosis in women. Sports Med 2005;35:779-830.

27. Matkovic V, Fontana D, Tominac C, Goel P, Chesnut CH $3^{\text {rd }}$. Factors that influence peak bone mass formation: A study of calcium balance and the inheritance of bone mass in adolescent females. Am J Clin Nutr 1990;52:878-88.

28. Rakhman L, Pangestika O, Fitriani D, Rudhanton R, Permatasari N. Aloe vera ethanol extract as a therapy for alveolar mandible regeneration in lipopolysaccharide-exposed rats. Int J App Pharm 2018;10:11-4.

29. Sivamaruthi B, Kesika P, Chaiyasut C. A review on anti-aging properties of probiotics. Int J App Pharm 2018;10:23-7. 